\title{
3 Research Square \\ Self-remedied Nanomedicine for Surmounting the Achilles's Heel of Photodynamic Tumor Therapy
}

Xiang Zhou

Southern Medical University

Jia-Qi Huang

Southern Medical University

Ling-Shan Liu

Southern Medical University

Fu-An Deng

Guangzhou Medical University

Yi-Bin Liu

Guangzhou Medical University

Yan-Mei Li

Southern Medical University

A-Li Chen

Guangzhou Medical University

Xi-Yong Yu

Guangzhou Medical University

Shi-Ying Li ( $\square$ lisy-sci@gzhmu.edu.cn )

Guangzhou Medical University https://orcid.org/0000-0003-0666-7634

Hong Cheng

Southern Medical University

Research Article

Keywords: Photodynamic therapy, HIF-1a, Hypoxia, Self-delivery, Self-assembly

Posted Date: June 3rd, 2021

DOI: https://doi.org/10.21203/rs.3.rs-580760/v1

License: (1) This work is licensed under a Creative Commons Attribution 4.0 International License.

Read Full License 


\section{Abstract}

Background: Photodynamic therapy (PDT) has been recognized as a promising tumor treatment for its minimal invasiveness, low side effects and on-demand light controllability. However, the oxygendependent PDT could exacerbate tumor hypoxia to upregulate the expression of hypoxia-inducible factor1a (HIF-1a), which would promote tumor growth and metastasis. Inhibition of HIF-1a activity is very necessary to PDT for effective tumor suppression.

Results: Herein, we developed a self-remedied nanomedicine based on a photosensitizer and a HIF-1a inhibitor to surmount the Achilles' heel of PDT for enhanced antitumor efficacy. Specifically, the nanomedicine (designated as CYC-1) was prepared by the self-assembly of Ce6 and YC-1 through $\pi-\pi$ stacking and hydrophobic interactions. Carrier-free CYC-1 held an extremely high drug loading rate, uniform size distribution and favorable stability. Compared with free Ce6, CYC-1 exhibited an improved cellular uptake behavior and an enhanced ROS production capability. Besides, CYC-1 had the overwhelming superiority on restraining tumor proliferation over the combined administration of Ce6 and YC-1. More importantly, CYC-1 preferred to accumulate in tumor tissue for efficient PDT by inhibiting the activity of HIF-1a. Ultimately, this YC-1-assistant PDT effectively restrained the tumor growth and caused a low system toxicity.

Conclusions: This carrier-free self-remedied strategy overcame the Achilles' heel of PDT on tumor suppression while induced a minimal side effect, which would expedite the development and clinical translation of nanomedicine for PDT against hypoxic tumors.

\section{Introduction}

As an emerging therapeutic modality in clinic, photodynamic therapy (PDT) has gained increasing attention nowadays for its unique advantages in tumor treatment [1-3]. It is well-known that PDT could induce cell apoptosis by utilizing photosensitizers to produce cytotoxic reactive oxygen species (ROS) in the presence of light and oxygen [4-6]. However, most photosensitizers are lipophilic compounds with a strong hydrophobicity and they are easy to aggregate in water [7-9]. In addition, the oxygen-dependent PDT would also restricted due to the hypoxic microenvironment of tumors [10-12]. Worse yet, the continued oxygen consumption by PDT would aggravate the tumor hypoxia, leading to the upregulation of hypoxia-inducible factor-1a (HIF-1a) [13-15]. Activated HIF-1 plays an important role in mediating adaptive responses to changes in tumor oxygenation, which could promote tumor growth and metastasis [16-18]. Moreover, the overexpressed HIF-1a has been confirmed to be associated with the treatment failure and increased patient mortality $[19,20]$. Thus, inhibition of HIF-1a activity is rather necessary to PDT for effective tumor suppression.

YC-1 (3-(5'-hydroxy-methyl-2'-furyl)-1-benzylindazole) has been widely recognized as a small molecular inhibitor of HIF-1a for tumor therapy [21-23]. Specifically, YC-1 could block the expression of HIF-1a at the post-transcriptional level so as to inhibit the transcription factor activity of HIF-1 [24]. Preclinical 
studies indicated that effective inhibition of HIF-1a would markedly impair tumor growth [25]. Furthermore, the downregulation of HIF-1a might sensitize tumors to PDT thereby improving the therapeutic effect [26-28]. It is supposed that the combination of YC-1 with PDT would significantly elevate the antitumor efficiency by HIF-1a inhibition. In view of the fact that different therapeutic agents hold diverse physicochemical properties and pharmacokinetics, construction of drug codelivery systems might be an effective strategy to realize their combined effect [29-32]. However, most of the traditional carrier-assistant drug delivery systems have some inevitable deficiencies including complex components, tedious preparation process and low drug loading rates [33,34]. What's more, the extra excipients may have the potential toxicity and induce adverse immune responses [35-37]. In this case, the emerging drug self-delivery systems (DSDSs), which consist of pure drugs without additional carriers, have suggested tremendous advantages and application potential in recent years $[38,39]$. Even so, it is still difficult to screen suitable drugs for preparing DSDSs and making them work in concert.16 Particularly, HIF-1a inhibitor-based DSDSs have rarely reported for enhanced PDT.

Herein, we developed a self-delivery nanomedicine based on YC-1 and the photosensitizer of Ce6 for enhanced photodynamic tumor therapy by HIF-1 a inhibition. As illustrated in Scheme 1A, Ce6 and YC-1 could self-assemble into the nanomedicine (designated as CYC-1) by virtue of $\pi-\pi$ stacking and hydrophobic interactions. Without extra carriers, CYC-1 possessed very high drug loading rate and it avoided the side effects caused by excipients. Besides, the intravenously administrated CYC-1 preferred to accumulate at the tumor site and then be internalized by tumor cells for effective drug delivery (Scheme 1B). More importantly, CYC-1 was demonstrated to downregulate the expression of HIF-1a in tumors thereby sensitizing the cells to PDT. As a result, in spite of the aggravated hypoxia by PDT, CYC-1 still significantly inhibited the tumor growth and induced a low systemic toxicity. This self-remedied strategy surmounted the Achilles' heel of oxygen-dependent PDT and decrease the resistance of tumors to PDT, which presented a novel paradigm on the development of nanomedicine for effective PDT against malignancies.

\section{Materials And Methods}

\section{Materials}

Chlorin e6 (Ce6) was purchased from Frontiersci. YC-1 (3-(5'-hydroxy-methyl-2'-furyl)-1-benzylindazole) was obtained from ApexBio. 2',7'-dichloroflorescein diacetate (DCFH-DA), singlet oxygen sensor green (SOSG), 3-(4,5-Dimethylthiazol-2-yl)-2,5-diphenyltetrazolium bromide (MTT), Hoechst 33342, CalceinAM/PI Live/Dead Viability/Cytotoxicity Kit and SDS-PAGE gel preparation kit were procured from Beyotime biotech. Co., Ltd. (China). Dulbecco's modified eagle's medium (DMEM), fetal bovine serum (FBS) and penicillin-streptomycin solution (PS) were purchased from Invitrogen Corp. Anti-HIF-1a antibody, anti-GAPDH antibody and corresponding secondary antibody were obtained from Abcam Corp.

\section{Cell culture}


Murine mammary carcinoma (4T1) cells were cultured in DMEM medium containing $10 \%$ FBS and $1 \%$ PS in an atmosphere with $5 \% \mathrm{CO}_{2}$ at $37^{\circ} \mathrm{C}$.

\section{Synthesis of CYC-1}

Firstly, $59.6 \mu \mathrm{L}$ of Ce6 and $30.4 \mu \mathrm{L}$ of $\mathrm{YC}-1$ (both dissolved in DMSO with a concentration of $10 \mathrm{~g} / \mathrm{L}$ ) were mixed and stirred for $4 \mathrm{~h}$ away from light. Subsequently, above solution was added into $3 \mathrm{~mL}$ of dd. water. The solution was ultrasounded for $0.5 \mathrm{~h}$ and stirred for $6 \mathrm{~h}$ afterwards. Then, the solution was dialyzed overnight (MWCO 1kDa) and centrifuged (3000 rpm) for $5 \mathrm{~min}$, so that the rest of free Ce6 and YC-1 as well as the aggregated Ce6 could be removed. The final product CYC-1 was obtained with 1:1 feed ratio by mass. Other formulations with different mass ratios of $\mathrm{Ce} 6$ and $\mathrm{YC}-1$ were synthesized with the same method.

\section{Characterizations}

The hydrodynamic diameters and zeta potential of CYC-1 were evaluated by Nano-Zetasizer Zen 3600 (Malvern). The morphology of CYC-1 was observed by transmission electron microscopy (TEM) using JEOL-1400 PLUS. UV-Vis absorbance spectra of Ce6, YC-1 and CYC-1 were determined by Evolution 300 (Thermo Scientific). High performance liquid chromatography (HPLC) were measured by SPD-M20A (SHIMADZU). Fluorescence spectra were characterized by Lumina (Thermo Scientific). Images of confocal laser scanning microscope (CLSM) were obtained and analyzed by LSM 880 (Carl Zeiss). H\&E staining of sectioned tissues was imaged by Eclipse $\mathrm{Ni}-U$ (Nikon). In vivo fluorescence images were obtained by In-Vivo FX PRO (BRUKER).

\section{In vitro singlet oxygen detection}

For fluorescence detection, SOSG was used to detect in vitro singlet oxygen by fluorescence spectrum. Briefly, SOSG $(5 \mu \mathrm{M})$ was incubated with CYC-1 $(2.37 \mathrm{mg} / \mathrm{L})$ or the equivalent concentration of Ce6 (2 $\mathrm{mg} / \mathrm{L})$ and $\mathrm{YC}-1(0.37 \mathrm{mg} / \mathrm{L})$ in PBS. Then, the solutions in light groups were exposed to light $(635 \mathrm{~nm}, 80$ $\mathrm{mW} / \mathrm{cm}^{2}$ ). The fluorescence intensity of each group was recorded every $10 \mathrm{~s}$ with an excitation wavelength of $488 \mathrm{~nm}$ and emission wavelength of $537 \mathrm{~nm}$. For CLSM observation, after seeded and incubated for $24 \mathrm{~h}, 4 \mathrm{~T} 1$ cells were washed with PBS and treated for $4 \mathrm{~h}$ with $\mathrm{CYC}-1$ ( $2.37 \mathrm{mg} / \mathrm{L})$ or the equivalent concentration of Ce6 $(2 \mathrm{mg} / \mathrm{L})$ and $Y C-1(0.37 \mathrm{mg} / \mathrm{L})$. After washed thrice, the cells were incubated with DCFH-DA $(10 \mu \mathrm{M})$ for $30 \mathrm{~min}$ and then subjected to irradiation $\left(1.8 \mathrm{~J} / \mathrm{cm}^{2}, 29.8 \mathrm{~mW} / \mathrm{cm}^{2}\right)$ for $30 \mathrm{~s}$. The fluorescence signals were imaged afterwards.

\section{Cellular uptake}

The cellular uptake behaviors of Ce6 and CYC-1 were assessed by CLSM and flow cytometry. 4T1 cells were seeded and incubated for $24 \mathrm{~h}$. Then, the culture medium was removed and the cells were treated with CYC-1 (5.93 mg/L) or the equivalent concentration of Ce6 (5 mg/L) for $0,2,4,8$ and $24 \mathrm{~h}$. To assess the cellular uptake performance, the intracellular fluorescence was observed by CLSM. Besides, the cells 
were also harvested and the cellular fluorescence was analyzed by flow cytometry for quantitative detection.

\section{MTT assay}

4T1 cells were seeded in 96-wells plates for incubation. After that, the cells were treated by Ce6, YC-1 or CYC-1 with gradient concentrations for $4 \mathrm{~h}$. Subsequently, 4T1 cells of irradiation groups were exposed to laser $\left(1.8 \mathrm{~J} / \mathrm{cm}^{2}, 29.8 \mathrm{~mW} / \mathrm{cm}^{2}\right)$ for $1 \mathrm{~min}$. Afterwards, the cells were incubated for another $20 \mathrm{~h}$. Then 20 $\mu \mathrm{L}$ of MTT $(5 \mathrm{~g} / \mathrm{L})$ was put into every well and incubated for $4 \mathrm{~h}$. Later, all the culture medium was removed and $150 \mu \mathrm{L}$ of DMSO was added to every well to dissolve the purple formazan. The plates were shook on the shaker at a low speed for $15 \mathrm{~min}$ and the absorbance at the particular wavelength $(570 \mathrm{~nm})$ of the solution was determined by microplate reader.

\section{Live/dead cell staining and cell apoptosis analysis}

For live/dead cell staining assay, 4T1 cells were seeded in glass bottom dish for incubation. Subsequently, the cells were treated with CYC-1 $(2.37 \mathrm{mg} / \mathrm{L})$ or the equivalent concentration of Ce6 (2 $\mathrm{mg} / \mathrm{L})$ and $\mathrm{YC}-1(0.37 \mathrm{mg} / \mathrm{L})$. After $4 \mathrm{~h}$, the plates were exposed to irradiation $\left(1.8 \mathrm{~J} / \mathrm{cm}^{2}, 29.8 \mathrm{~mW} / \mathrm{cm}^{2}\right)$ for $30 \mathrm{~s}$ or kept in the dark. Then all of the cells were incubated for another $20 \mathrm{~h}$. After washed with PBS twice, the cells were stained with calcein-AM/PI for 15 min and washed twice again. The cellular fluorescence was observed by CLSM. For cell apoptosis analysis, the cells were treated with CYC-1, Ce6 or YC-1 using the similar method. After irradiated for $20 \mathrm{~s}$ and incubated for $20 \mathrm{~h}$, the cells were washed with PBS and stained with Annexin V-FITC/PI. Finally, the cells were harvested and the cellular fluorescence was analyzed by flow cytometry for apoptotic detection.

\section{Western blot analysis}

4T1 cells were seeded and incubated with CYC-1 $(5.93 \mathrm{mg} / \mathrm{L})$ or the equivalent concentration of Ce6 (5 $\mathrm{mg} / \mathrm{L})$ and $\mathrm{YC}-1(0.93 \mathrm{mg} / \mathrm{L})$ for $2 \mathrm{~h}$. Then the cells were incubated for $2 \mathrm{~h}$ under hypoxia environment. Subsequently, the cells were irradiated by laser $\left(1.8 \mathrm{~J} / \mathrm{cm}^{2}, 29.8 \mathrm{~mW} / \mathrm{cm}^{2}\right)$ for $10 \mathrm{~s}$ or kept in the darkness. After further incubation for $20 \mathrm{~h}$, the cells were cooled on ice and thoroughly washed by PBS thrice. Ice cooled RIPA solution with two enzyme inhibitors was then added into the cells for cell lysis (30 $\mathrm{min})$. The cell lysate solution was collected and centrifuged $\left(12,000 \mathrm{rpm}, 20 \mathrm{~min}, 4{ }^{\circ} \mathrm{C}\right)$ to remove unbroken cells, nuclei and cell fragments. The separated proteins were quantified by BCA protein Assay Kit and normalized to the same concentration. After that, the samples were separated by electrophoresis using SDS-PAGE gel and transferred to a PVDF membrane which was blocked in TBST with $5 \%$ skim milk for $1 \mathrm{~h}$ afterwards. The membrane was then incubated with the rabbit anti-HIF-1 alpha or the rabbit antiGAPDH primary antibody at $4{ }^{\circ} \mathrm{C}$ overnight. Next, the HRP conjugated goat anti-rabbit secondary antibody was incubated with the membrane for $2 \mathrm{~h}$ at RT. The PVDF membrane was washed by TBST thrice. Finally, the western lightning ECL substrate was loaded to the membrane under dark and the fluorescence was monitored by enhanced chemiluminescence exposor. 


\section{In vivo fluorescence imaging}

The in vivo experiments were conducted according to the guidelines of animal experiments established by Laboratory Animal Center of Southern Medical University (Guangzhou, China) as well as the Regulations of Guangdong Province on the Administration of Experimental Animals. In vivo tumor model was established by subcutaneous injection of $4 T 1$ cells into the female BalB/C mice. The $4 T 1$ tumorbearing mice were administrated with CYC-1 $(2.07 \mathrm{mg} / \mathrm{kg})$ by intravenous injection. At the preset time, the mice were imaged by small animal imaging system. After $12 \mathrm{~h}$, the mice were sacrificed to collect the heart, liver, spleen, lung, kidney and tumors for ex vivo fluorescence imaging.

\section{Immunofluorescence staining}

Immunofluorescence staining was conducted to examine the expression of HIF-1a protein after treatment. Briefly, 4T1 tumor-bearing mice were administrated with CYC-1 $(2.07 \mathrm{mg} / \mathrm{kg})$ or the equivalent concentration of $\mathrm{Ce} 6(1.75 \mathrm{mg} / \mathrm{kg})$ or YC-1 $(0.32 \mathrm{mg} / \mathrm{kg})$ by intravenous injection. $4 \mathrm{~h}$ later, the mice in light groups were irradiated $\left(635 \mathrm{~nm}, 80 \mathrm{~mW} / \mathrm{cm}^{2}\right)$ for $10 \mathrm{~min}$. After another $20 \mathrm{~h}$, the mice were sacrificed. Tumor tissues were collected and sliced to tumor frozen sections. The slices were incubated with rabbit anti-HIF-1 alpha primary antibody containing $3 \%$ BSA at $4{ }^{\circ} \mathrm{C}$ overnight. Afterwards, the Alexa Fluor 594 labeled secondary antibody was added for $1 \mathrm{~h}$ incubation. The cell nuclei were stained with DAPI for 20 min later. Finally, the tumor sections were imaged by CLSM.

\section{In vivo anti-tumor study}

4T1 tumor model was established as described above. Subsequently, the tumor-bearing mice were divided into 6 groups randomly (7 mice for each group) for the administration of CYC-1 $(2.07 \mathrm{mg} / \mathrm{kg})$ or the equivalent concentration of Ce6 $(1.75 \mathrm{mg} / \mathrm{kg})$ or YC-1 $(0.32 \mathrm{mg} / \mathrm{kg})$ by intravenous injection. $4 \mathrm{~h}$ later, the mice in light groups were irradiated $\left(635 \mathrm{~nm}, 80 \mathrm{~mW} / \mathrm{cm}^{2}\right)$ for $10 \mathrm{~min}$. The tumor volume $(\mathrm{V})$ and body weight of each mouse were recorded every other day for 2 weeks. $V$ was defined as following formula: $V=\left(\right.$ length $\times$ width $\left.^{2}\right) / 2$. On day 15 , the mice were sacrificed to collect the major organs (heart, liver, spleen, lung, kidney) and tumors for further tissue section and hematoxylin and eosin (H\&E) staining. The blood sample was also collected from the mice for further blood routine and blood biochemical examination. The photography of each section was performed using a Nikon upright microscope.

\section{Results And Discussion}

\section{Preparation and characterization of CYC-1}

CYC-1 was prepared by the self-assembly of Ce6 and YC-1 via an amended nanoprecipitation method. To obtain an ideal nanomedicine, the self-assembly process of Ce6 and $\mathrm{YC}-1$ was studied at different feed ratios. As shown in Fig. 1A, TEM observations indicated that Ce6 and YC-1 at the feed ratio of 1:1 could fabricate into spherical nanoparticles with relatively uniform size and morphology. Furthermore, the 
particle size distribution was evaluated by DLS. As displayed in Fig. 1B, at the feed ratio of 1:1, the harvested nanomedicine had the minimum average size and its distribution was regular. Subsequently, the assembly mechanism was explored by using the UV-Vis spectrum. After treated with $\mathrm{NaCl}$, no obvious changes were found on the shapes of the UV-vis spectra (Fig. 1C), which suggested an ignorable intermolecular electrostatic interaction in CYC-1. However, different spectra of CYC-1 were observed when dissolved in water and DMSO (Fig. 1D). Particularly, CYC-1 held a broader and red-shifted Soret band of Ce6 in aqueous phase, which indicated $\pi-\pi$ stacking and hydrophobic interactions between Ce6 and YC-1. What's more, the addition of hydrophobic SDS significantly impacted the spectrum of CYC-1 (Fig. 1E), demonstrating that SDS participated in the assembly of CYC-1. Based on these, it was speculated that the $\pi-\pi$ stacking and hydrophobic interactions might be the main drivers of drug self-assembly. The drug loading rates of $\mathrm{Ce} 6$ and $\mathrm{YC}-1$ were confirmed to be $84.3 \%$ and $15.7 \%$ by using UV-vis spectrum (Fig. S1) and high performance liquid chromatography (HPLC) (Fig. S2). Afterwards, the stability of CYC-1 was also evaluated. As shown in Fig. 1F, the particle size and PDI of CYC-1 were detected to have no obvious fluctuations in one week. The favorable stability of DSDS was the precondition for biomedical applications. Taken together, by virtue of $\pi-\pi$ stacking and hydrophobic interactions, Ce6 and YC-1 at proper feed ratio could assemble into the nanomedicine with a uniform morphology and size distribution as well as favorable dispersity and stability (Fig. 1G).

\section{Evaluation of cellular uptake and ROS generation}

After successful preparation of CYC-1, its cellular uptake behavior was evaluated by CLSM and flow cytometry. As displayed in Fig. 2A, with the prolonged incubation time, 4T1 cells treated with either Ce6 or CYC-1 exhibited a gradually enhanced red fluorescence, which demonstrated an incubation timedependent cellular uptake. However, compared with Ce6, CYC-1 could be internalized more effectively with a much stronger red fluorescence in $4 \mathrm{~T} 1$ cells. Similar results were confirmed by the quantitative fluorescence analysis using flow cytometry (Fig. 2B). No matter incubation for 2, 4, 8 or $24 \mathrm{~h}$, the fluorescence intensity of $4 \mathrm{~T} 1$ cells treated with $\mathrm{CYC}-1$ was more than 5 times as high as that of treated with Ce6. These results indicated that the self-assembled nanomedicine gained the superiority on cellular drug delivery over the hydrophobic photosensitizer.

Furthermore, the ROS production ability of CYC- 1 was also detected to assess its potential for PDT. Above all, the ROS generated by Ce6 and CYC-1 was monitored by fluorescence spectrum using SOSG as the ${ }^{1} \mathrm{O}_{2}$ probe. As shown in Fig. $2 \mathrm{C}$, in the absence of light, both Ce6 and $\mathrm{CYC}-1$ failed to produce ${ }^{1} \mathrm{O}_{2}$. Once exposed to light, the SOSG fluorescence dramatically increased, indicating abundant production of ${ }^{1} \mathrm{O}_{2}$. Subsequently, the cellular ROS level was measured by CLSM using DCFH-DA as the ROS sensor. As described in Fig. 2D, after treated with CYC-1 and light, 4T1 cells presented bright green fluorescence, which implied lots of ROS production. Of special note, although $\mathrm{Ce} 6$ in solution could generate abundant ROS, it was unable to elevate the ROS level of tumor cells under light irradiation. Consistent conclusion was obtained by the mean fluorescence intensity (MFI) analysis (Fig. 2E). Compared with the PBS group, only a little fluorescence enhancement was found in 4T1 cells treated with Ce6 and light, which should be ascribed to the limited cellular internalization of hydrophobic Ce6 (Fig. 2A). The main reason for it should 
be the limited cellular internalization of hydrophobic Ce6 (Fig. 2A). To sum up, this self-delivery strategy could significantly improve the cellular uptake of therapeutic agents and promote the ROS production in cells for effective photodynamic tumor therapy.

\section{Cytotoxicity of CYC-1}

Prior to detecting the antitumor performance, the cytotoxicity of CYC-1 was investigated against 4T1 cells via MTT assay. As indicated in Fig. 3A, in the absence of light irradiation, Ce6, YC-1 and CYC-1 had such a low toxicity that the cell viability was higher than $85 \%$. However, after irradiated with light, Ce6 exhibited an obviously enhanced cytotoxicity due to the initiation of PDT. Such striking differences also illustrated the controllability of PDT in tumor therapy. More importantly, in comparison with the PDT of Ce6, CYC-1 with light showed a much more robust antitumor effect. One of the main reasons should be the improved cellular uptake of therapeutic agents after self-assembly. Another primary reason might be that the YC-1 mediated HIF-1a inhibition sensitized the tumor cells to PDT, leading to an increased cytotoxicity. Besides, it should be noted that CYC-1 could restrain the cell proliferation more effectively than the combined administration of $\mathrm{Ce} 6$ and $\mathrm{YC}-1$ in the presence of light. This result highlighted again the advantage of the self-remedied nanomedicine in tumor therapy.

Subsequently, the anti-proliferation ability was further evaluated by detecting the cell apoptosis using flow cytometry. As shown in Fig. 3B, when without illumination, the percentages of viable cells were more than $96 \%$ in all groups. The limited PDT of Ce6 could only cause less than half the tumor cells apoptosis. However, with the assistance of YC-1, the PDT efficacy of Ce6 was amplified that over $60 \%$ of the cells were found in early or late apoptotic state. More interestingly, there were almost no living cells after treated with CYC-1 and light, which demonstrated the best antitumor effect owing to the HIF-1a inhibition and self-delivery strategy. In addition, the cells after different treatments were performed for live/dead staining analysis by CLSM (Fig. 3C). Particularly, 4T1 cells treated with CYC-1 and light showed bright red fluorescence and a dash of green fluorescence, verifying that the overwhelming majority of the cells had been dead after the YC-1-assistant PDT.

To validate the inhibitory effect of YC-1 on HIF-1a, 4T1 cells after various treatments were carried out for western blot analysis. Notably, previous investigations had confirmed that the self-delivery strategy of CYC-1 could significantly improve the cellular uptake and ROS production of Ce6 (Fig. 2). In this case, the cells treated with CYC-1 should consume more oxygen for PDT than those treated by $\mathrm{Ce} 6$ in the presence of light. However, the most expression of HIF-1a was actually observed on the cells treated by Ce6 and light (Fig. S3), which indicated the maximal oxygen consumption. Moreover, after simultaneously treated with YC-1, the PDT of Ce6 induced HIF-1a activation was found to be suppressed. Based on these, it could be deduced that YC-1 could really inhibit HIF-1a to surmount the Achilles' heel of PDT for enhanced antitumor efficiency.

\section{Antitumor study in vivo}


Antitumor study of CYC-1 was performed under light irradiation against the 4T1 tumor-bearing mice (Fig. 4A). After intravenous administration, the biodistribution of CYC-1 was investigated by fluorescence imaging using small animal imaging system. As illustrated in Fig. 4B, the injected CYC-1 initially distributed throughout the body with the blood flow. Subsequently, a preferential fluorescence aggregation was found on the tumor site, which implied an effective tumor accumulation of CYC-1 via EPR effect. $12 \mathrm{~h}$ after injection, the mouse was sacrificed to collect the tumor tissue and main organs for ex vivo imaging. Obviously, in addition to the main metabolic organ of liver, tumor exhibited the strongest fluorescence accumulation, which further demonstrated the EPR-mediated passive targeting ability of CYC-1.

Inspired by it, the antitumor efficacy of CYC-1 was then evaluated in vivo after intravenous injection. As shown in Fig. 4C, the tumor growth was monitored every other day. The mice in PBS group without any treatments were used as the control, whose tumors grew very fast. Besides, YC-1 and CYC-1 had a certain ability of tumor suppression while the latter exhibited a better effect. The possible reason should be that the nanosized CYC-1 could be accumulated into tumor tissues more effectively to inhibit the HIF-1a activity and impair tumor growth. More importantly, in the presence of light, CYC-1 had absolute superiority over $\mathrm{Ce} 6$ on the tumor inhibition, which should be attributed to the improved drug delivery efficiency after self-assembly as well as the enhanced PDT efficacy through HIF-1a inhibition. Besides, the body weights of the mice were increasing during the treatments (Fig. 4D), which indicated a low system toxicity of these therapeutic agents. At the end of treatments, the tumor tissues were obtained for photographing (Fig. 4E) and weighing (Fig. 4F), which further verified the best antitumor effect of CYC-1 under light irradiation. Moreover, the tumor tissues were also stained by H\&E for histological examination. Compared with other groups, the tumors treated with CYC-1 and light held lots of damaged cells without nucleus (Fig. 4G), illustrating that the YC-1 assistant PDT could maximally destroy the tumor tissue to restrain its malignant proliferation.

In order to deeply explore the mechanism of $\mathrm{YC}-1$, the tumor tissues were carried out for immunofluorescent staining after various treatments. As reflected in Fig. $4 \mathrm{H}$, the immunofluorescence of HIF-1a in YC-1 and CYC-1 groups was weaker relative to that in PBS group, which confirmed the HIF-1a inhibition mediated by YC-1. Conversely, the tumor tissue after the PDT of Ce6 showed an obviously enhanced immunofluorescence, suggesting that the PDT aggravated the tumor hypoxia and caused the upregulation of HIF-1a. Even so, CYC-1 with light was found to not increase the expression of HIF-1a. This significant difference strongly demonstrated that $\mathrm{YC}-1$ as a competent inhibitor of HIF-1a could effectively mitigate the tumor hypoxia to improve the therapeutic effect of PDT. The self-remedied strategy of CYC-1 was capable of surmounting the Achilles' heel of PDT, suggesting a great potential in hypoxic tumor therapy.

\section{Biosafety analysis of CYC-1}

Favorable biosafety is necessary for therapeutic agents in biomedical applications. Although nanomedicine could realize the targeted drug delivery through EPR effect, it is inevitable to have some 
aggregation on normal tissues. To assess the biosafety of $\mathrm{CYC}-1$ in vivo, the main organs of the mice after treatments were sliced and stained by H\&E. As shown in Fig. 5A, the histological morphologies of these organs were observed to have no obvious variations, which implicated a low side effect. The major reasons for it should be that the PDT of CYC-1 had a good regioselectivity under local irradiation and it was less toxic when without light exposure. Moreover, the serum biochemical indexes of blood urea nitrogen (BUN), alanine aminotransferase (ALT), aspartate transaminase (AST) and uric acid (UA) were detected to have no significant abnormality (Fig. 5B), which suggested little damage of therapeutic agents to liver and kidney. Additionally, blood routine analysis of the mice was also carried out (Fig. 5C). Relevant parameters were basically in normal scopes, confirming a favorable blood compatibility of CYC1. Undoubtedly, CYC-1 held good antitumor performance with few adverse reactions.

\section{Conclusion}

In summary, a carrier-free self-remedied nanomedicine was developed to surmount the Achilles' heel of PDT for effective tumor suppression. By adjusting the feed ratio, Ce6 and YC-1 successfully assembled into the nanomedicine of $\mathrm{CYC}-1$ with a high drug loading rate and uniform size distribution. Besides, CYC1 had a good dispersibility and stability in aqueous phase regardless of the strong hydrophobicity of Ce6 and YC-1. Moreover, compared with free Ce6, CYC-1 exhibited an improved cellular uptake behavior, which was of benefit to generate more ROS in the presence of light irradiation. Besides, CYC-1 displayed much better antitumor effect in comparison with the combined administration of Ce6 and YC-1 which suggested the advantages of this self-delivery strategy for tumor suppression. Regardless of the fact that the oxygen-dependent PDT could exacerbate the hypoxia to cause a decreasing therapeutic efficacy, the YC-1-assistant PDT was demonstrated to significantly inhibit the tumor growth by downregulating the expression of HIF-1a. This carrier-free self-remedied strategy overcame the Achilles' heel of PDT and leaded to a minimal side effect, which would expedite the development and clinical translation of nanomedicine for PDT against hypoxic tumors.

\section{Declarations}

\section{Acknowledgements}

Not applicable.

\section{Author's contributions}

$\mathrm{HC}$ was responsible for the manuscript revision. $\mathrm{SL}$ and $\mathrm{XZ}$ designed the experiments. JH, LS, FD, YL and $Y L$ performed the experiments. $X Y$ and $A C$ commented on this manuscript. All authors read and approved the final manuscript.

\section{Funding}


This work was supported by the National Natural Science Foundation of China $(51803086,52073140$, 51802049), the Guangdong Basic and Applied Basic Research Foundation (2021A1515010418, 2021B1515020043), the Science and Technology Programs of Guangzhou (202002030178, 201904010324) and the Young Elite Scientist Sponsorship Program by CAST (2018QNRC001).

\section{Availability of data and materials}

All data generated or analyzed during this study are included in this manuscript and its supplementary material

\section{Ethics approval and consent to participate}

The in vivo experiments were conducted according to the guidelines of animal experiments established by Laboratory Animal Center of Southern Medical University (Guangzhou, China) as well as the Regulations of Guangdong Province on the Administration of Experimental Animals.

\section{Consent for publication}

No individual person's data were included in this research.

\section{Competing interests}

Authors declare there are no competing interests, financial and non-financial, in relation to the work described.

\section{References}

1. Agostinis P, Berg K, Cengel KA, Foster TH, Girotti AW, Gollnick SO, et al. Photodynamic therapy of cancer: an update. CA-Cancer J Clin. 2011;61:4.

2. Chen JM, Fan TJ, Xie ZJ, Zeng QQ, Xue P, Zheng TT, et al. Advances in nanomaterials for photodynamic therapy applications: status and challenges. Biomaterials. 2020;237:119827.

3. Park J, Lee YK, Park IK, Hwang SR. Current limitations and recent progress in nanomedicine for clinically available photodynamic therapy. Biomedicines. 2021;9:1.

4. Yang BW, Chen Y, Shi JL. Reactive oxygen species (ROS)-based nanomedicine. Chem Rev. 2019;119:8.

5. Luby BM, Walsh CD, Zheng G. Advanced photosensitizer activation strategies for smarter photodynamic therapy beacons. Angew Chem Int Edit. 2019;58:9.

6. Nguyen VN, Yan YX, Zhao JZ, Yoon JY. Heavy-atom-free photosensitizers: from molecular design to applications in the photodynamic therapy of cancer. Accounts Chem Res. 2021;54:1.

7. Lan MH, Zhao SJ, Liu WM, Lee CS, Zhang WJ, Wang PF. Photosensitizers for photodynamic therapy. Adv Healthc Mater. 2019;8:13. 
8. Tong XN, Gan SJ, Wu JH, Hu YQ, Yuan A. A nano-photosensitizer based on covalent organic framework nanosheets with high loading and therapeutic efficacy. Nanoscale. 2020;12:13.

9. Ghosh S, Carter KA, Lovell JF. Liposomal formulations of photosensitizers. Biomaterials. 2019;218:119341.

10. Zhou Z, Song J, Nie L, Chen X. Reactive oxygen species generating systems meeting challenges of photodynamic cancer therapy. Chem Soc Rev. 2016;45:23.

11. Li X, Kwon N, Guo T, Liu Z, Yoon J. Innovative strategies for hypoxic-tumor photodynamic therapy. Angew Chem Int Ed. 2018;57:36.

12. Kumari R, Sunil D, Ningthoujam RS. Hypoxia-responsive nanoparticle based drug delivery systems in cancer therapy: an up-to-date review. J Control Release. 2020;319:135.

13. Fan WP, Huang P, Chen XY. Overcoming the Achilles' heel of photodynamic therapy. Chem Soc Rev. 2016;45:23.

14. Phung CD, Tran TH, Pham LM, Nguyen HT, Jeong JH, Yong CS, Kim JO. Current developments in nanotechnology for improved cancer treatment, focusing on tumor hypoxia. J Control Release. 2020;324:413.

15. Mitra S, Cassar SE, Niles DJ, Puskas JA, Frelinger JG, Foster TH. Photodynamic therapy mediates the oxygen-independent activation of hypoxia inducible factor 1 alpha. Mol Cancer Ther. 2006;5:12.

16. Rankin EB, Giaccia AJ. Hypoxic control of metastasis. Science. 2016;352:175.

17. Harris AL. Hypoxia-a key regulatory factor in tumour growth. Nat Rev Cancer. 2002;2:38.

18. Papandreou I, Cairns RA, Fontana L, Lim AL, Denko NC. HIF-1 mediates adaptation to hypoxia by actively downregulating mitochondrial oxygen consumption. Cell Metab. 2006;3:3.

19. Petrova V, Annicchiarico-Petruzzelli M, Melino G, Amelio I. The hypoxic tumour microenvironment. Oncogenesis. 2018;7:20.

20. Masoud GN, Li W. HIF-1 a pathway: role, regulation and intervention for cancer therapy. Acta Pharm Sin B. 2015;5:5.

21. Bhattarai $D, X u X Z$, Lee K. Hypoxia-inducible factor-1 (HIF-1) inhibitors from the last decade (2007 to 2016): A"structure-activity relationship" perspective. Med Res Rev. 2018;38:4.

22. Kong J, Kong FD, Gao J, Zhang QB, Dong SY, Gu F, et al. YC-1 enhances the anti-tumor activity of sorafenib through inhibition of signal transducer and activator of transcription 3 (STAT3) in hepatocellular carcinoma. Mol Cancer. 2014;13:7.

23. Zhang B, Huang XC, Wang HS, Gou SH. Promoting antitumor efficacy by suppressing hypoxia via nano self-assembly of two irinotecan-based dual drug conjugates owning a HIF-1 alpha inhibitor. J Mater Chem B. 2019;7:35.

24. Lang JY, Zhao X, Wang XC, Zhao Y, Li YY, Zhao RF, et al. Targeted co-delivery of the iron chelator deferoxamine and a HIF1a inhibitor impairs pancreatic tumor growth. ACS Nano. 2019;13:2.

25. Semenza GL. Targeting HIF-1 for cancer therapy. Nat Rev Cancer. 2003;3:10. 
26. Liu P, Xie X, Shi XY, Peng Y, Ding JS, Zhou WH. Oxygen-self-supplying and HIF-1a-inhibiting core-shell nanosystem for hypoxia-resistant photodynamic therapy. ACS Appl Mater Interfaces. 2019;11:51.

27. Broekgaarden M, Weijer R, Krekorian M, van den IJssel B, Kos M, Alles LK, et al. Inhibition of hypoxiainducible factor 1 with acriflavine sensitizes hypoxic tumor cells to photodynamic therapy with zinc phthalocyanine-encapsulating cationic liposomes. Nano Res. 2016;9:6.

28. Zhang ZT, Wang RY, Huang XX, Luo RJ, Xue JW, Gao J, et al. Self-delivered and self-monitored chemo-photodynamic nanoparticles with light-triggered synergistic antitumor therapies by downregulation of HIF-1a and depletion of GSH. ACS Appl Mater Interfaces. 2020;12:5.

29. Tibbitt MW, Dahlman JE, Langer R. Emerging frontiers in drug delivery. J Am Chem Soc. 2016;138:3.

30. Guo Q, Jiang C. Delivery strategies for macromolecular drugs in cancer therapy. Acta Pharm Sin B. 2020;10:6.

31. Zhou FY, Feng B, Yu HJ, Wang DG, Wang TT, Ma YT, et al. Tumor microenvironment-activatable prodrug vesicles for nanoenabled cancer chemoimmunotherapy combining immunogenic cell death induction and CD47 blockade. Adv Mater. 2019;31:14.

32. Yu FZ, Tu YL, Luo SW, Xiao X, Yao W, Jiang ML, et al. Dual-drug backboned polyprodrug with a predefined drug combination for synergistic chemotherapy. Nano Lett. 2021;21:5.

33. P Yuan, GL Fan, LP Zhao, LS Liu, FA Deng, XY Jiang, et al. Tumor targeted self-synergistic nanoplatforms for arsenic-sensitized photodynamic therapy. Acta Biomater. 2020;117:349.

34. Wang S, Liu X, Chen SZ, Liu ZR, Zhang XD, Liang XJ, et al. Regulation of $\mathrm{Ca}^{2+}$ Signaling for drugresistant breast cancer therapy with mesoporous silica nanocapsule encapsulated doxorubicin/siRNA cocktail. ACS Nano. 2019;13:1.

35. Wang CP, Li L, Zhang S, Yan Y, Huang Q, Cai XP, et al. Carrier-free platinum nanomedicine for targeted cancer therapy. Small. 2020;16:49.

36. Huang L, Zhao SJ, Fang F, Xu T, Lan MH, Zhang JF. Advances and perspectives in carrier-free nanodrugs for cancer chemo-monotherapy and combination therapy. Biomaterials. 2021;268:120557.

37. Zhao LP, Zheng RR, Huang JQ, Chen XY, Deng FA, Liu YB, et al. Self-delivery photo-immune stimulators for photodynamic sensitized tumor immunotherapy. ACS Nano. 2020;14:12.

38. Qin SY, Zhang AQ, Cheng SX, Rong L, Zhang XZ. Drug self-delivery systems for cancer therapy. Biomaterials. 2017;112:234.

39. Zhao LP, Zheng RR, Chen HQ, Liu LS, Zhao XY, Liu HH, et al. Self-delivery nanomedicine for $\mathrm{O}_{2}{ }^{-}$ economized photodynamic tumor therapy. Nano Lett. 2020;20:3.

\section{Figures}


A
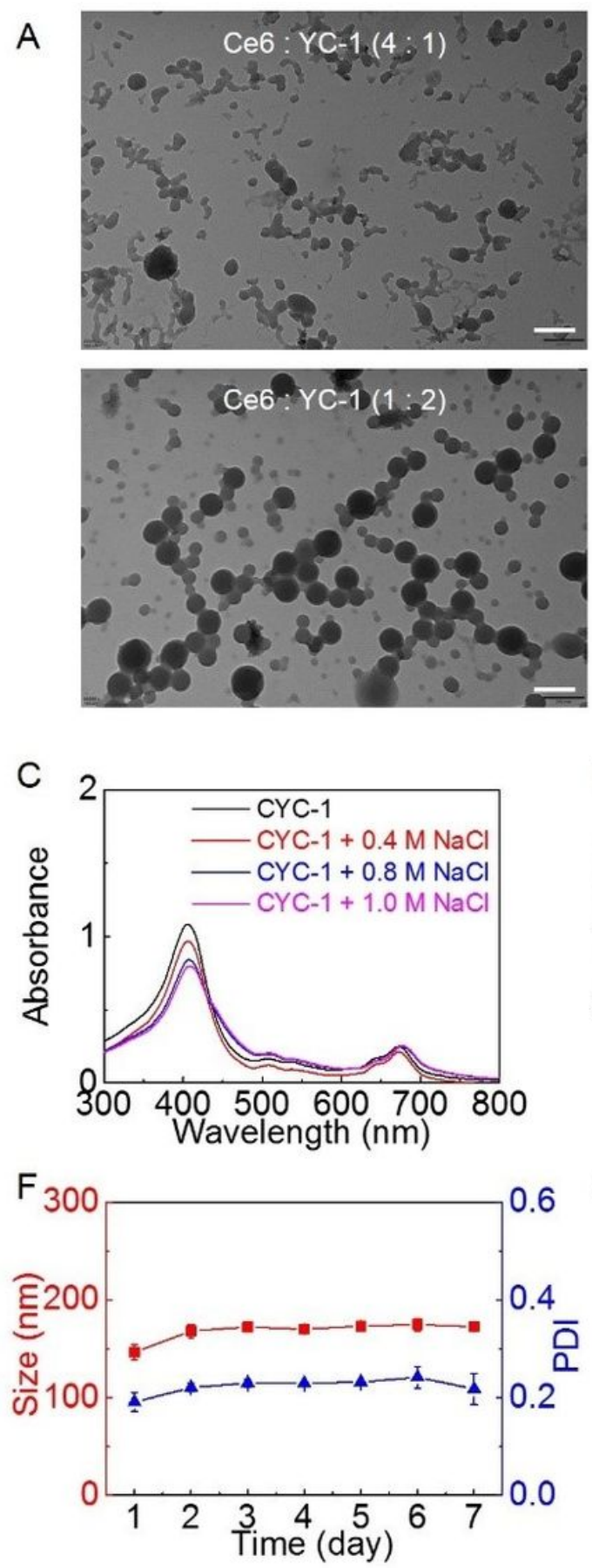
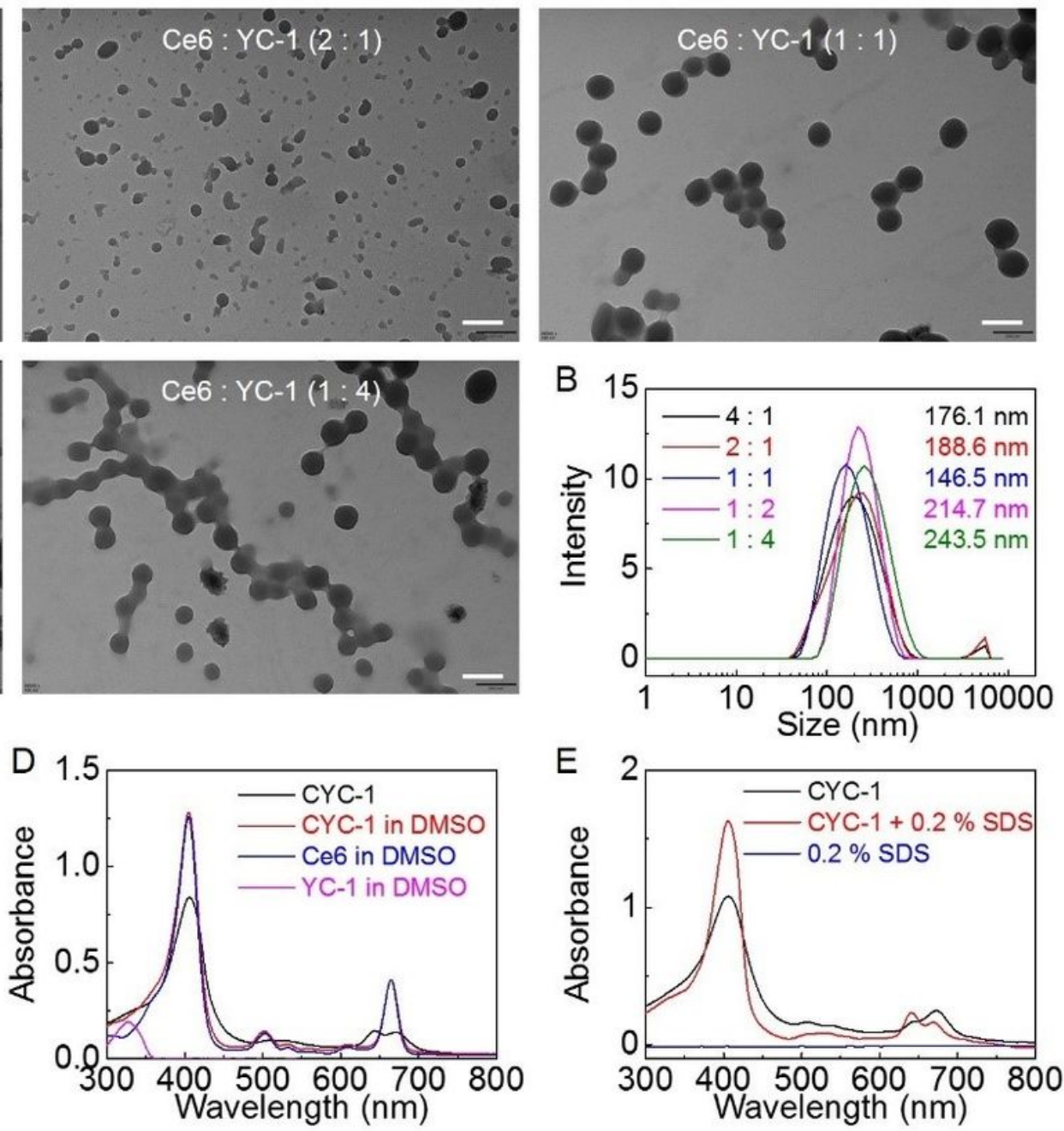

G
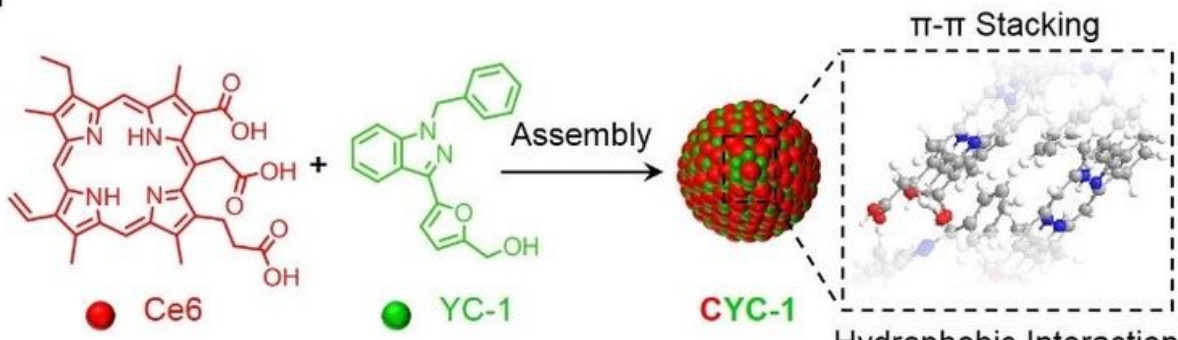

Hydrophobic Interaction

\section{Figure 1}

Preparation and characterization of CYC-1. (A) TEM images and (B) the particle size distributions of the self-assembled nanomedicine with the feed ratios of Ce6 and YC-1 at $4: 1,2: 1,1: 1,1: 2$ or $1: 4$. Scale bar: $200 \mathrm{~nm}$. (C) UV-vis absorbance of CYC-1 in NaCl solutions (0.2 M, 0.4 M or 0.8 M). (D) UV-vis absorbance of Ce6, YC-1 and CYC -1 in the distilled water or in DMSO. (E) UV-vis absorbance of CYC-1 in the presence or absence of $0.2 \%$ SDS. (F) The particle size distribution and PDI changes of CYC-1 in 7 days. (G) The proposed mechanism of the preparation of CYC-1 by the self-assembly of Ce6 and YC-1 through $\pi-\pi$ stacking and hydrophobic interactions. 

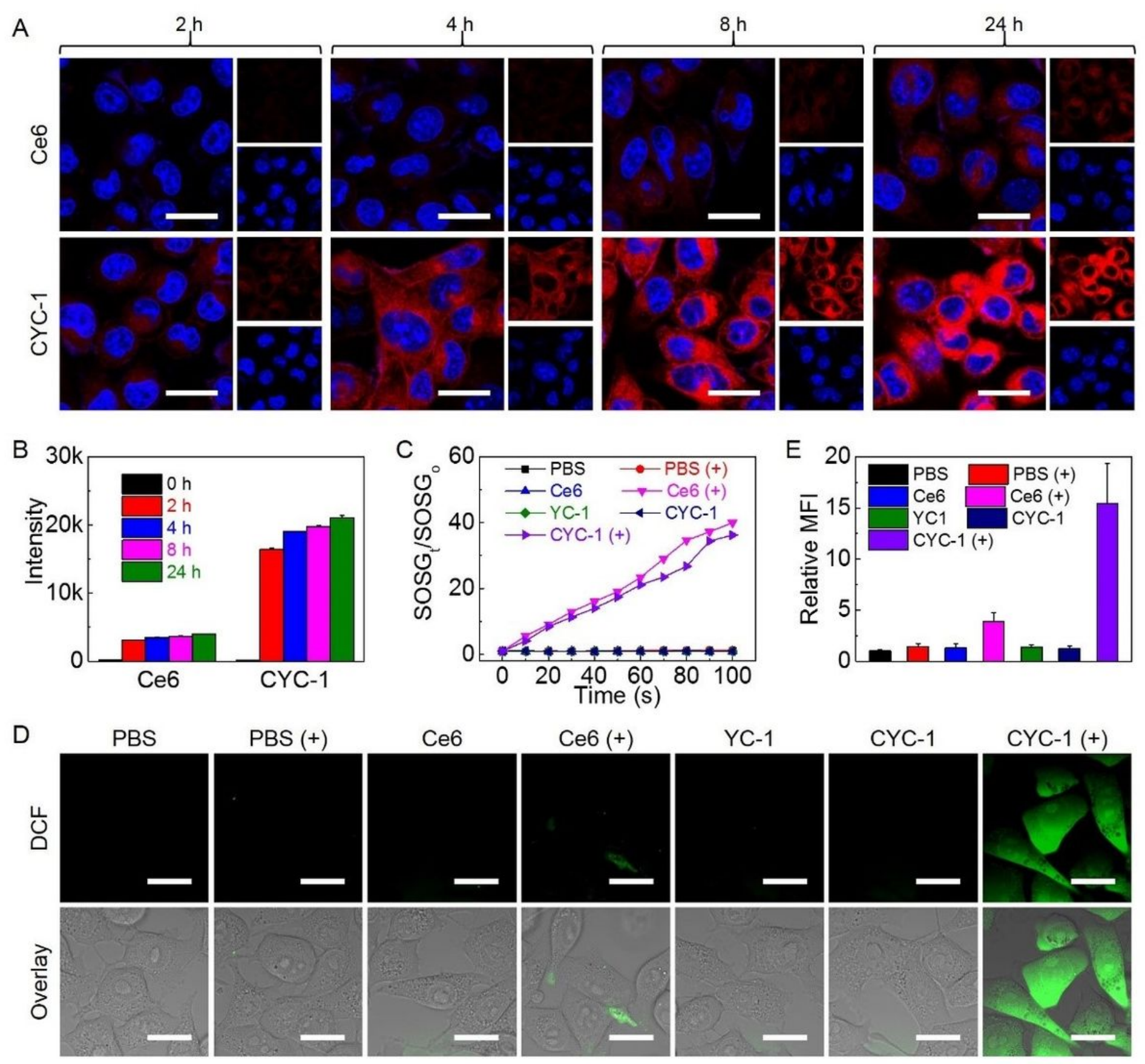

Figure 2

Cellular uptake behaviors and ROS production ability of CYC-1. (A) CLSM images and (B) flow cytometry analysis of the fluorescence in 4T1 cells after treatment with Ce6 and CYC-1 for 2, 4, 8 or $24 \mathrm{~h}$. Scale bar: $20 \mu \mathrm{m}$. (C) 102 production abilities of Ce6 and CYC-1 when with or without light irradiation. SOSG was employed as the 102 probe. (D) CLSM images and (E) relative mean fluorescence intensity (MFI) analysis of $4 \mathrm{~T} 1$ cells after treatment with Ce6, YC- 1 or CYC-1 in the presence or absence of light. DCFH-DA was used as the ROS probe. Scale bar: $20 \mu \mathrm{m}$. "+" represented the addition of light irradiation. 
A

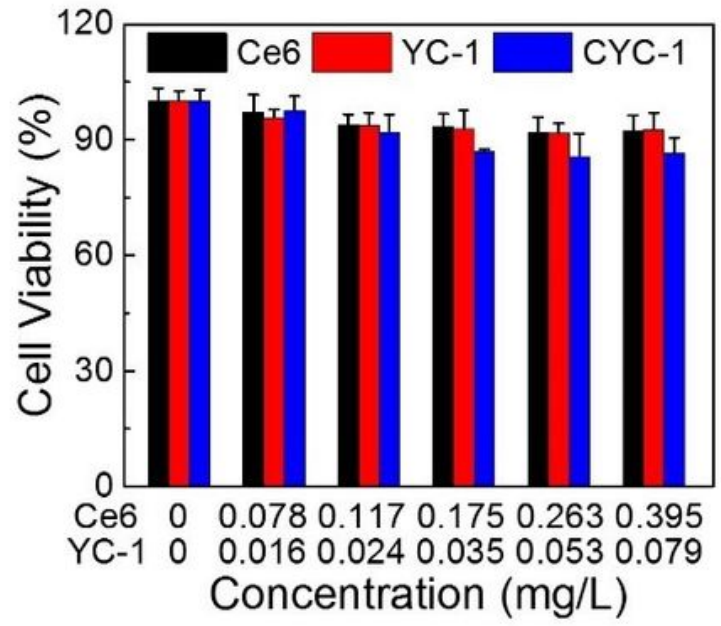

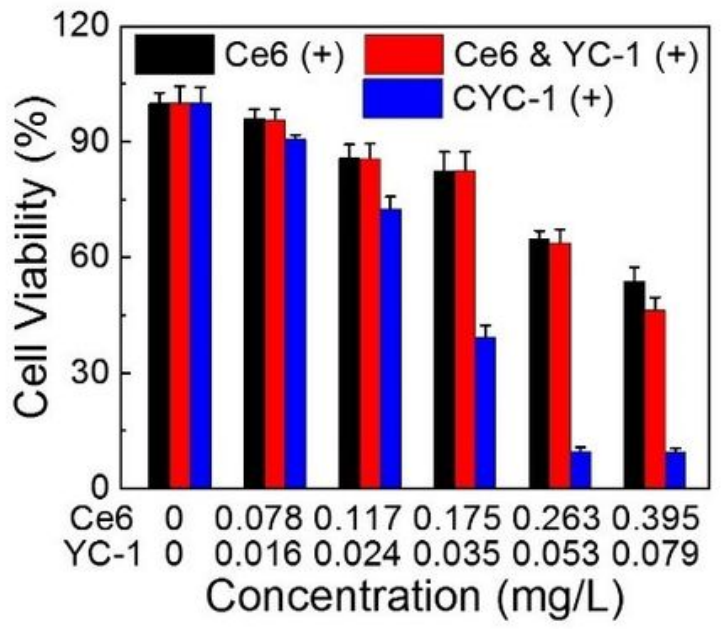

B
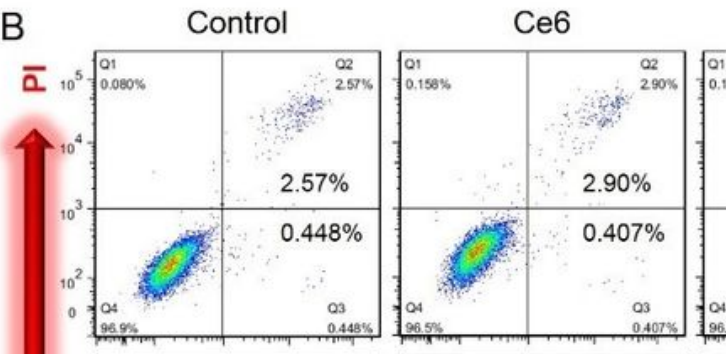

YC-1 Ce6 \& YC-1

CYC-1
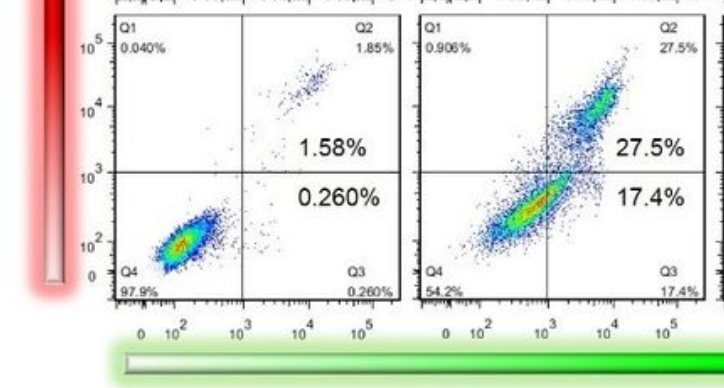

$\sqrt{\log 2 x}$
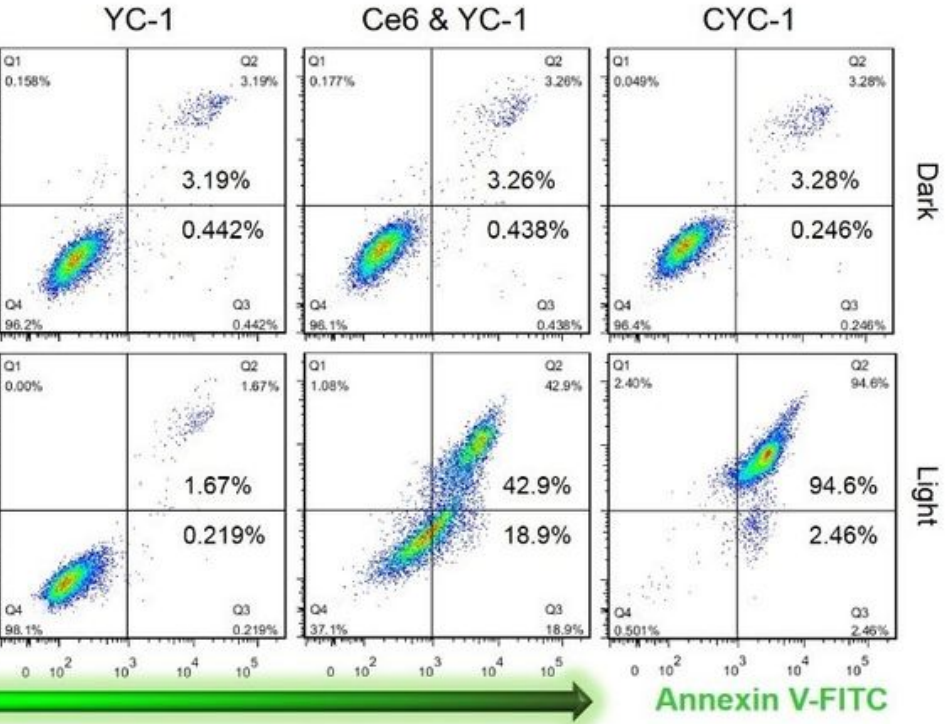

C

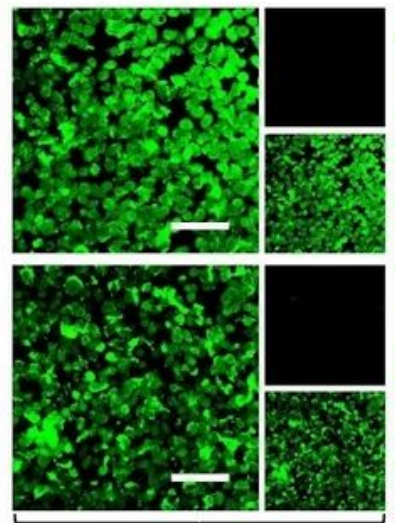

Control

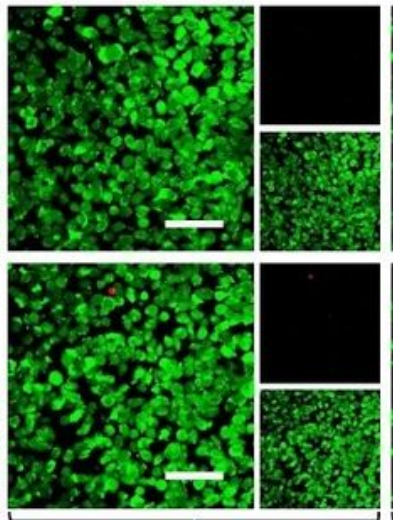

Ce6

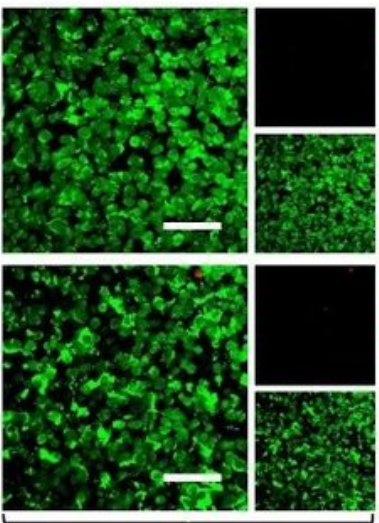

Ce6 \& YC-1

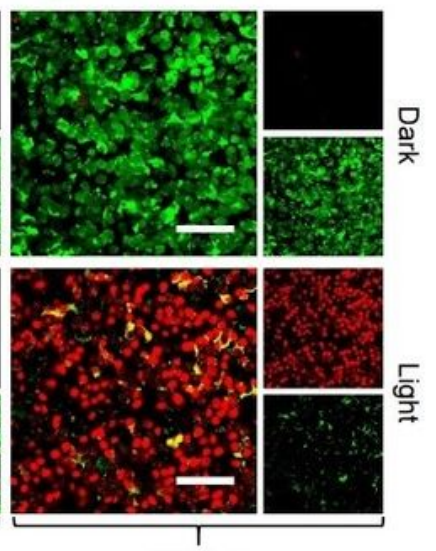

CYC-1

Figure 3

Anti-proliferation ability of CYC-1. (A) 4T1 cell viability detection by MTT assay, (B) 4T1 cell apoptosis analysis by flow cytometry and (C) live/dead cell staining analysis of 4T1 cells by CLSM after treatment with Ce6, YC-1, Ce6 \& YC-1, or CYC-1 in the absence or presence of light. Scale bar: $100 \mu \mathrm{m}$. " +" represented the addition of light irradiation. 

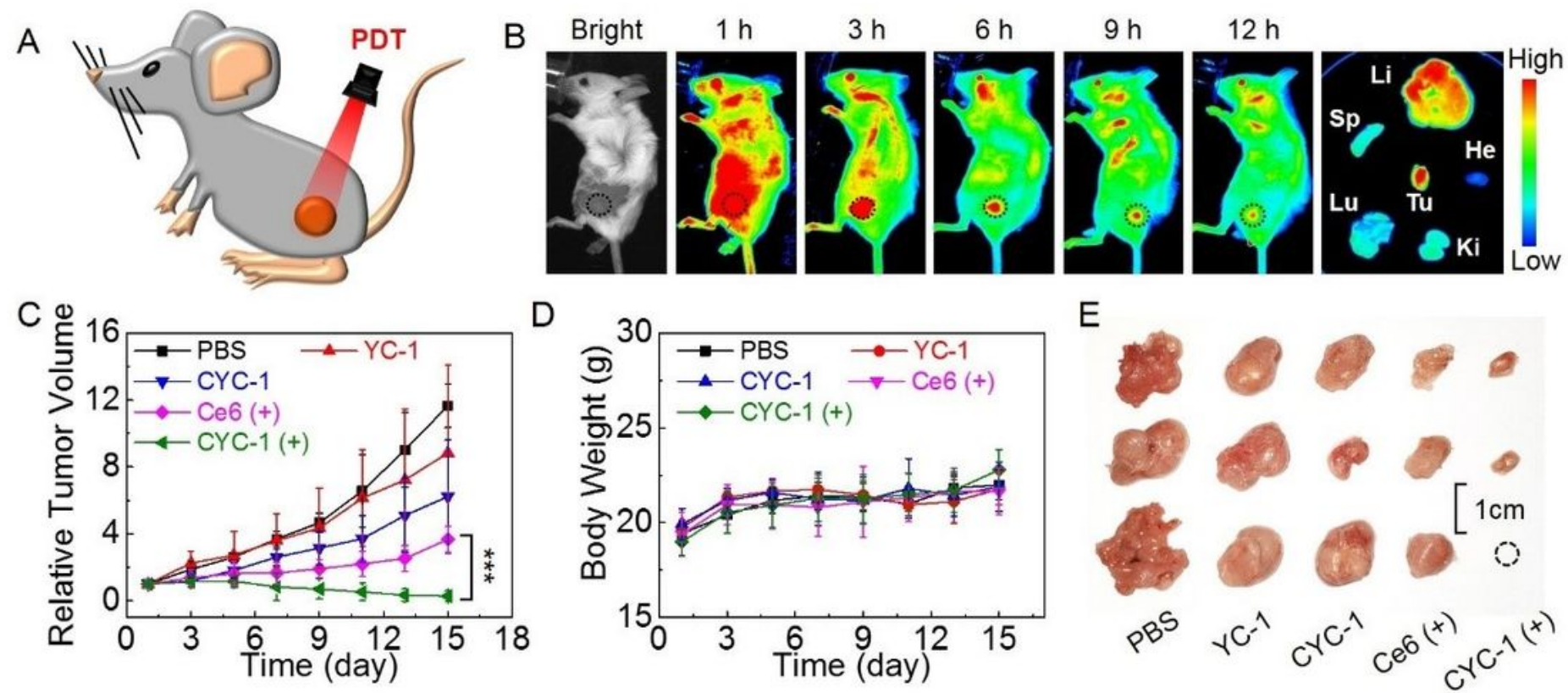

$\mathrm{D}$

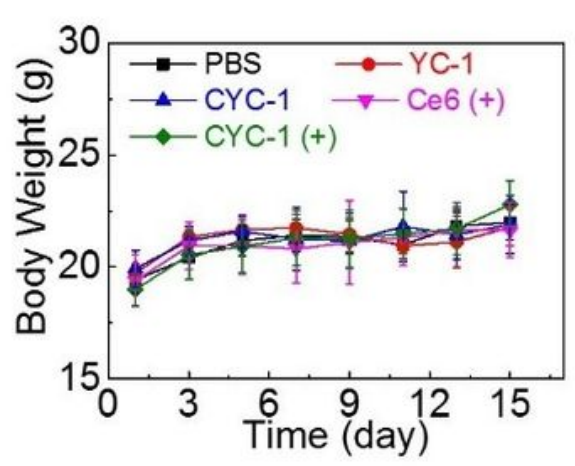

$\mathrm{E}$
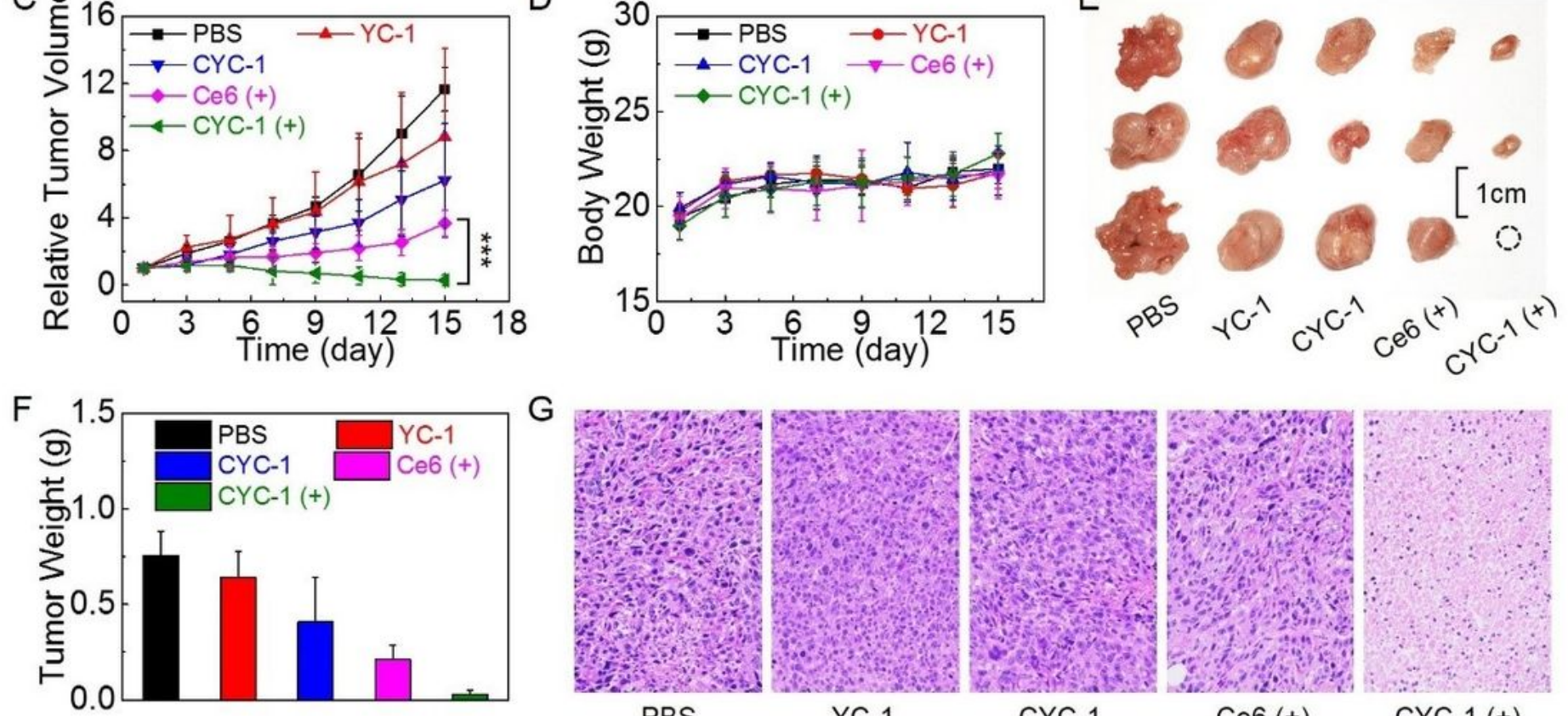

G
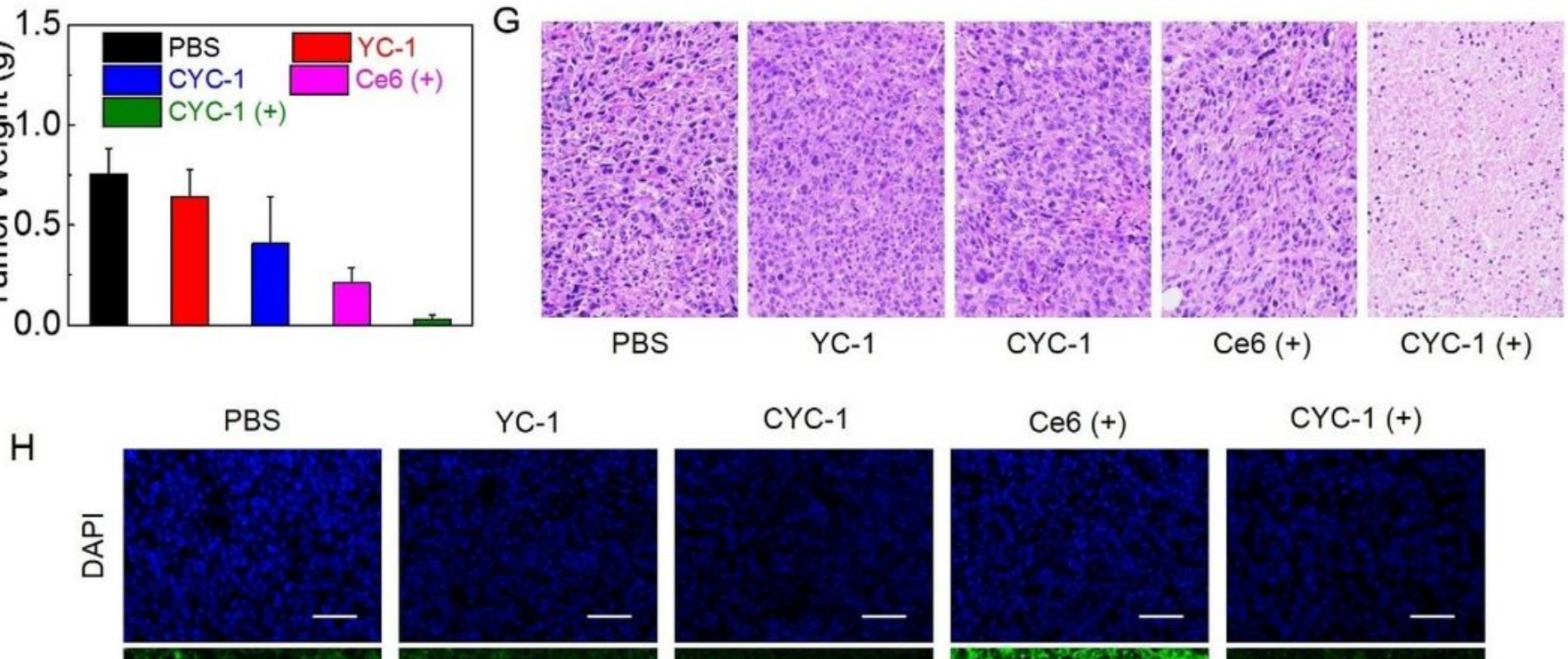

CYC-1 (+)
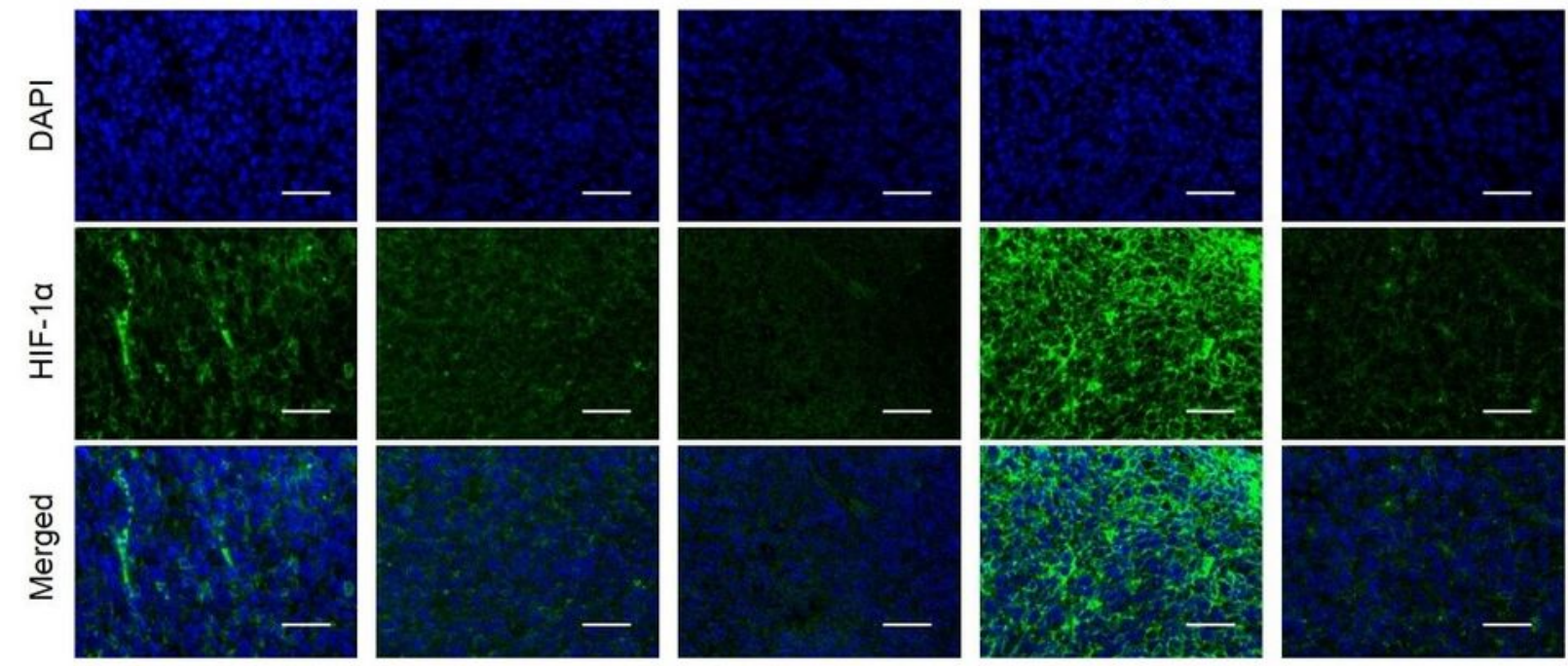

\section{Figure 4}

Anti-tumor study in vivo. (A) Schematic illustration of the anti-tumor study of CYC-1 under light irradiation against 4T1 tumor-baring mouse. (B) Real time fluorescence images of the tumor-bearing mouse as well as ex vivo fluorescence images of sacrificed organs and tumor tissue after intravenously injection with CYC-1. In which, He, Li, Sp, Lu, Ki and Tu represented heart, liver, spleen, lung, kidney and tumor respectively. (C) The relative tumor volume and (D) body weight changes of the mice after administration 
with Ce6, YC-1 or CYC-1 in the presence or absence of light in 15 days. (E) The images of represented tumor tissues, $(F)$ the mean tumor weight and $(G)$ H\&E staining of tumor tissues at the end of treatments. (H) Immunofluorescent staining of tumor tissues after treatment with Ce6, YC-1 or CYC-1 for $24 \mathrm{~h}$ in the presence or absence of light. Scale bar: $50 \mu \mathrm{m}$. "+" represented the addition of light irradiation.

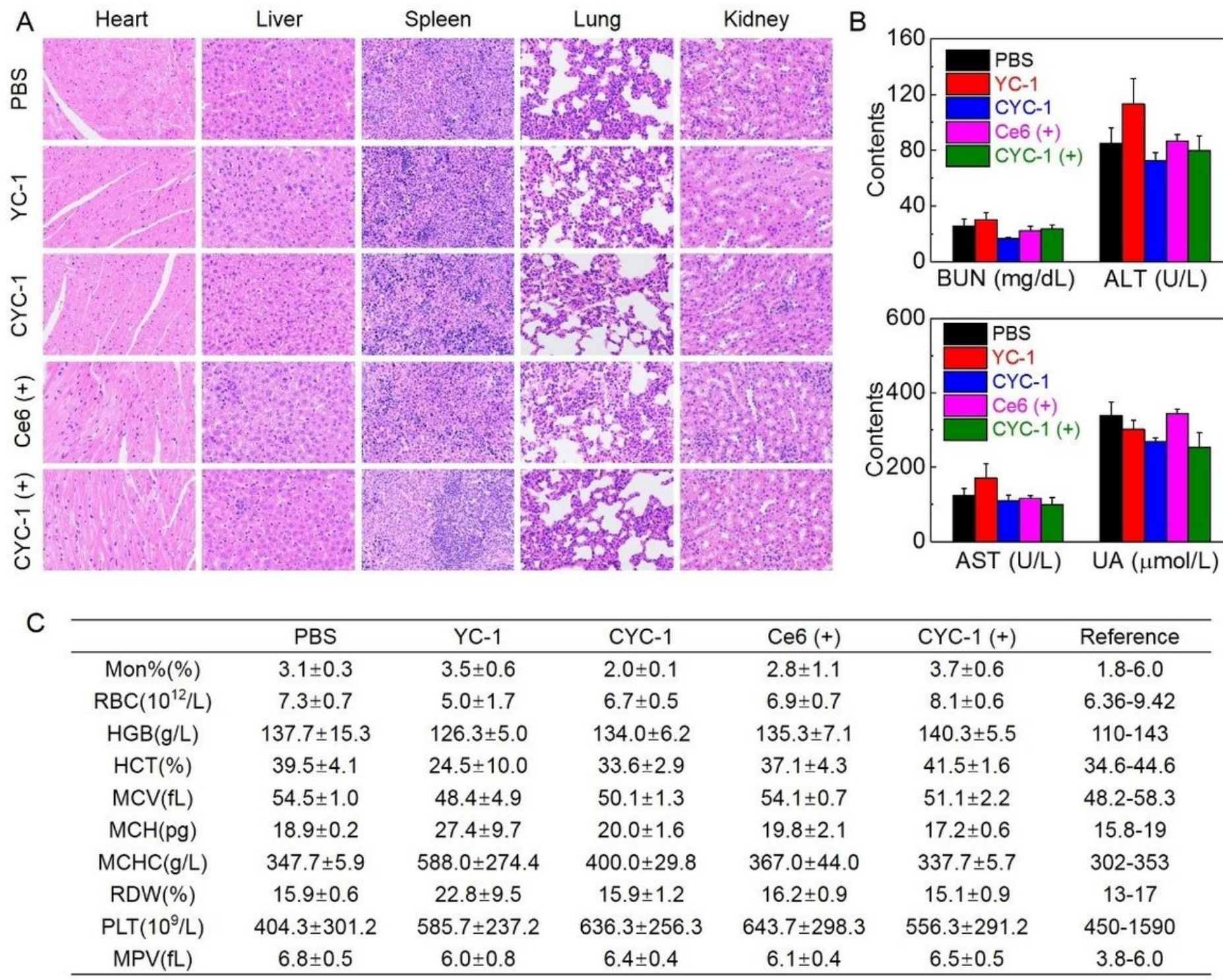

\section{Figure 5}

Biosafety analysis of CYC-1. (A) H\&E staining of the heart, liver, spleen, lung and kidney after treatment with Ce6, YC-1 or CYC-1 in the presence or absence of light. (B) Biochemical analysis of BUN, ALT, AST, UA and (C) blood routine analysis of the mice after treatment with Ce6, YC-1 or CYC-1 in the presence or absence of light. "+" represented the addition of light irradiation.

\section{Supplementary Files}

This is a list of supplementary files associated with this preprint. Click to download. 
- Scheme1.jpg

- Supportinglnformation.doc 